\title{
Seasonal Cycles in Testicular Activity, Gonadotropin, and Thyroxine in the Painted Turtle, Chrysemys picta, under Natural Conditions
}

\author{
Paul Licht, ${ }^{*}$ Gary L. Breitenbach,$\dagger$ and Justin D. Congdon $\dagger$ \\ *Department of Zoology, University of California, Berkeley, California 94720, and †Museum of Zoology and \\ Division of Biological Sciences, The University of Michigan, Ann Arbor, Michigan 48109
}

Accepted October 16, 1984

\begin{abstract}
Plasma levels of testosterone ( $\mathrm{T})$, thyroxine $\left(\mathrm{T}_{4}\right)$ and follicle-stimulating hormone (FSH) were measured in a ficld population of the painted turtle, Chrysemys picta, in Michigan. All three hormones showed pronounced seasonality; plasma luteinizing hormone (LH) remained nondetectable. Plasma T and FSH concentrations were highly correlated and exhibited biphasic cycles with peaks in spring and fall, whereas $\mathrm{T}_{4}$ showed a single peak in summer, coincident with the nadir in T. Hormone levels were minimal on the day that animals emerged from hibernation at the end of March; T and FSH then increased rapidly (in ca. 1 week after emergence) to a transient peak that persisted for only ca. 2 weeks. Plasma T and FSH rose again in September before the onset of hibernation and shortly after the late summer peak in spermatogenetic recrudescence. Plasma $T_{4}$ increased more gradually after emergence in spring and did not peak for 1-2 months, coincident with the nadir in T. The profile in plasma FSH is consistent with the view that this gonadotropin may regulate androgen secretion but it did not show the expected relationship to the spermatogenetic cycle. Separate studies indicated that the turtles are sensitive to capture and handling; hormone levels (especially androgen) fall precipitously within a day of capture. These "stress" effects may account for discrepancies with previous studies of plasma $T$ in this species. 1985 Academic Press. Inc.
\end{abstract}

Data on the endocrine changes associated with the annual testicular cycle of turtles are somewhat conflicting. Early histological studies in temperate-zone species indicated that the Leydig cells are most conspicuously developed in spring when the testes are minimal in size and spermatogenically inactive, while these presumptive androgen-secreting tissues are atrophied in late summer when the testes otherwise appear to be most active (Moll, 1979). Such a dissociation between androgen secretion and spermatogenesis has been taken as evidence for the differential secretion of two distinct gonadotropins that control the two aspects of testicular activity, but physiological studies of the chelonian gonadotropins have not provided evidence for such a duality of function of the two pituitary gonadotropins (e.g., Licht, 1979). Unfortunately, there are no data on plasma gonadotropin levels during the annual testicular cycle and the few direct measurements of plasma androgen in turtles have not been entirely consistent with the concept of a temporal separation of androgen secretion and spermatogenesis (reviewed by Licht, 1982).

A seasonal study of plasma androgen in the painted turtle Chrysemys picta, indicated that androgen secretion was highest in the spring when testes were otherwise regressed (Callard et al., 1976); Ganzhorn and Licht (1983) also reported that plasma testosterone was higher in spring than fall. However, results of experimental studies in this species were inconsistent with these findings: testis growth and spermatogenesis could be stimulated at any time of year by exposure to warm temperatures and this recrudescence was invariably accompanied by elevated plasma androgen, even though 
Leydig cells did not show typical histological activation (Ganzhorn and Licht, 1983). Thus, one would expect plasma androgens to be elevated in fall when testes are enlarged, a situation that would also be predicted by available physiological data on gonadotropin action in turtles, since each gonadotropin tends to affect both aspects of testicular function (Licht, 1979). Indeed, recent studies of the subtropical Chrysemys dorbigni revealed a single seasonal peak in androgen coincident with maximal testicular growth (Silva et al., 1984).

One possible explanation for the discrepancies between seasonal and experimental observations in $C$. picta is that the former were based largely on commercially collected animals that had variable (and generally undefined) histories. Thus, either unseasonal conditions encountered in captivity or the "stress" effects of capture and captivity may have altered normal seasonal patterns of androgen levels (cf. Licht et al., 1983).

Besides uncertainties regarding the fundamental relationship between spermatogenetic and androgen-secreting activities of the chelonian testis, other factors may complicate the interpretation of the relationship between gonadotropins and various testicular activities. For example, avian studies have identified a reciprocal functional relationship between seasonally high circulating thyroxine $\left(\mathrm{T}_{4}\right)$ and androgen titers; each may modulate the level of the other, and $\mathrm{T}_{4}$ may also block gonadotropin stimulation of androgenic secretion by the testis (Jallageas and Assenmacher, 1979). A similar situation was suggested by the seasonal patterns of $\mathrm{T}_{4}$ and gonadotropin in the cobra (Bona-Gallo et al., 1980). On the other hand, in green sea turtles, Chelonia mydas, pronounced androgen cycles occurred in the absence of a thyroxine cycle (Licht et al., 1985). Comparative data for reptiles are too limited to allow generalizations about this feature of the annual en- docrine cycles; there are no data on the seasonal relationship between the thyroid and testicular cycles in temperate-zone turtles.

The present investigation took advantage of an intensive field study on a natural population of painted turtles (Tinkle et al., 1981) to reexamine the seasonal cycle in plasma androgen in free-living animals and to determine its relationship to pituitary gonadotropins and the thyroidal cycle. Additional tests were undertaken to evaluate the problem of capture and handling on these hormonal levels.

\section{MATERIALS AND METHODS}

Study population. This investigation was conducted in conjunction with an ecological field study of the painted turtle at the E. S. George Reserve of the University of Michigan, Livingston County, Michigan. Details of this study and methods of capturing turtles are discussed in Tinkle et al. (1981) and Congdon and Tinkle (1982). Adult turtles were captured by drift fences which intercepted their movements on land, by baited or basking traps in the water, or opportunistically by hand. Drift fences and basking traps were checked daily and turtles would have been confined in the traps for a maximum of about $10 \mathrm{hr}$. Baited traps were checked less frequently and turtles may have been in them for up to 2 days. In all cases, blood samples were taken (from the tip of the tail) within a few minutes of first handling the turtle. A total of 128 individual turtles were sampled during this study (4 of these were recaptured one or more times) to yield 139 blood seasonal samples. Studies of postcapture effects consisted of placing animals in aquatic pens for 1-6 days after initial sampling; 29 individuals were used in these tests ( 8 in spring, 16 in fall, and the others scattered throughout summer).

The study was begun in the fall (September) 1981, in the month before the animals entered winter hibernation. A sample of 12 adult males was captured as they emerged from the mud on the day (March 28) that the ice cover was just beginning to break up in a creek near the main study area. Previous experience has shown that the turtles emerge from this site about a week or more before most of those that would be included in the remainder of the study. Hence, turtles captured in the main study area starting April 15 (Fig. 2) had probably only emerged about a week or less earlier. Sampling continued until the end of the activity season in 1982. Minimum and maximum water temperatures recorded at a depth of $1 \mathrm{~m}$ are shown for the main portion of the second ycar in Fig. 1; animals tend 


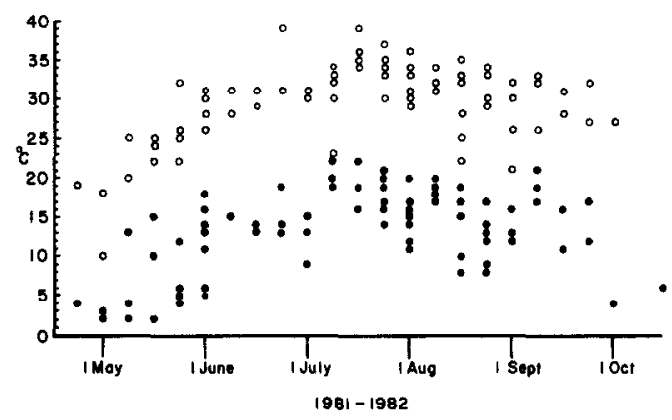

FIG. 1. Minimum (shaded circles) and maximum (open circles) water temperatures measured at a depth of $1 \mathrm{~m}$ every third day at the major study site where turtles were trapped in Michigan. All values for a week are shown at the same time. Water still contained ice when the turtles began emerging from hibernation at the bottom of ponds in early April.

to emerge on the first warm days after the ice cover begins to break up; thus, water temperatures would warm gradually from near freezing during April.

Hormone analyses. Heparinized blood samples were centrifuged and the plasma was frozen until analyzed. Testosterone $(T)$ and thyroxine $\left(T_{4}\right)$ were measured by radioimmunoassay (RIA) as detailed in Licht et al. (1984); ether extraction was employed for the steroid analysis. Luteinizing hormone (LH) was measured by a sea turtle LH $\beta$-RIA system (Licht et al., 1977; Licht, 1978).

Follicle-stimulating hormone (FSH) was measured in plasma by a RIA with a rabbit antiserum raised against $C$. mydas FSH and a homologous tracer and standard (see Licht and Papkoff, 1985 for details on this FSH). This RIA showed some cross-reaction with LH, but inhibition slopes for LH were consistently nonparallel (low) to FSH. In tests to validate its use for this heterologous species, cross-reaction with parallel inhibition slopes was demonstrated with many $C$. picta pituitary extracts and plasma samples. Incubation medium from pituitaries incubated with LHRH showed parallel cross-reaction with FSH; this immunoactivity was distinctive from secreted $\mathrm{LH}$ in gel filtration - the FSH immunoactivity behaved as a larger molecule than $\mathrm{LH}$ as expected from fractionation studies. Additional evidence for the applicability of this RIA to heterologous chelonian species came from the demonstration of parallelism with a highly purified heterologous chelonian FSH (from snapping turtle). Also, elevated immunoactivity was observed with plasma from castrate turtles, Sternotherus odoratus, even though LH often remained undetectable in these. Because of some low nonspecific reactions with plasma, values below $4 \mathrm{ng} / \mathrm{ml}$ were considered as nondetectable. This potential LH cross-reaction was not considered important here since plasma samples showed parallelism and LH was not detectable in any of the samples. All samples were tested in a single assay to reduce variability.

Statistical analyses were performed with a microcomputer program, Microstat (Ecosoft, Inc.). Oneway analysis of variance was used to test for differences among the nine biweekly periods and Duncan's multiple range test was used to identify differences between individual periods; log transformation of plasma $\mathrm{T}$ was employed. Regression analyses and ANOVA were used to test correlations.

\section{RESULTS}

Figure 2 presents individual plasma $\mathrm{T}$ values for the 1982 season to show the distribution of collecting dates and to illustrate the relatively rapid changes that occur after the animals become active in spring. To facilitate discussion of seasonal trends, data were arbitrarily pooled into bimonthly intervals (from the 1st to 15 th and from the 16 th to the end of each month). Mean values for all three hormones measured are presented in Fig. 3. ANOVA revealed highly significant differences among the resulting nine sampling periods for all three hormones: for T, $F_{(8,136)}=12.34, P<$ 0.0001 ; for FSH, $F_{(8,124)}=7.56, P<0.0001$; for $\left.\mathrm{T}_{4}, F_{(8,132)}=13.255, P<0.0001\right)$. Individual levels of plasma $\mathrm{T}$ and $\mathrm{FSH}$ were

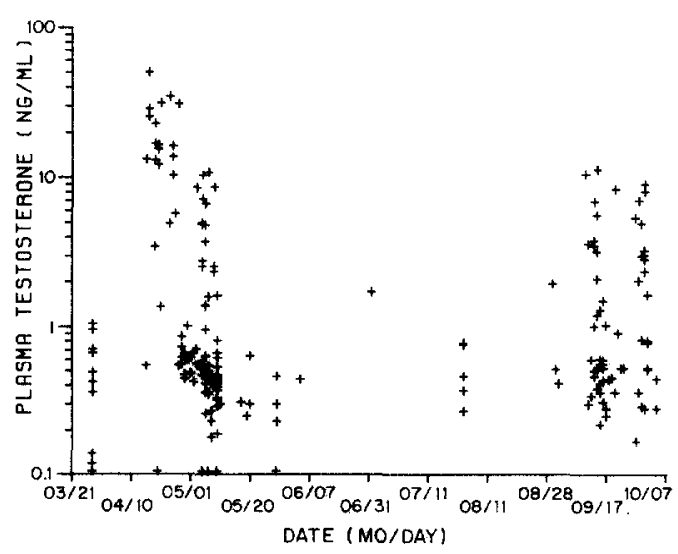

FIG. 2. Individual plasma testosterone values by day of collection for the 1982 study season. The first samples (on March 28) were taken from turtles captured on the day they emerged from hibernation. 
highly correlated for the entire study $(r=$ $\left.0.64, F_{(1,128)}=88.5 ; P<0.0001\right)$. Within biweekly periods, a significant correlation was observed only in April when the two hormones were highest and most variable $\left(F_{(1,19)}=3.678, P=0.0017\right)$. Plasma $\mathrm{T}_{4}$ was not correlated with the other two hormones. None of the hormones correlated with water temperature.

All hormones were uniformly low in the newly emerging animals in late March, but they were markedly elevated in most of those collected after April 15 (Figs. 2-3). Plasma T and FSH showed a transient peak; they remained elevated (in the population) for only about 2 weeks and showed a precipitous decline after about May 1. By contrast, plasma $\mathrm{T}_{4}$ continued to rise pregressively and did not peak until late May, almost 2 months after emergence. The extent of this peak could not be accurately assessed because of the lack of data for June, but $\mathrm{T}_{4}$ levels had clearly declined by late July (one animal collected in June had minimal levels but a second was still elevated to near peak levels). The peak in plasma $T_{4}$ coincided with the complete suppression of the spring rise in the reproductive hormones.

Plasma $T_{4}$ continued to decline until the end of the activity season; hence, a single scasonal peak was evident. However, both $\mathrm{T}$ and FSH showed a second increase during September; this elevation over minimal summer values (by about 10 -fold) was evident in both years. Although mean values were not as high in fall as in spring, a multiple-range test indicated that the mean values for the April peak and September (1981 and 1982) samples did not differ, but both were significantly higher $(P$ $<0.05$ ) than in early June and late July.

\section{Postcapture Effects}

Plasma levels of all three hormones were typically depressed by holding the animals for a day or more after the initial sample, and plasma $T$ appeared to be the most sen- sitive. Individual examples are illustrated in Fig. 4, and the mean levels of plasma $T$ for the 8 animals tested in spring and 16 in fall over a 3-day period are shown in Fig. 5. When the hormone levels were near seasonal minimum at time of capture, there was no discernible change over the next 14 days of holding. In the 24 cases where $T$ values were elevated at the time of capture, all but 2 showed a precipitous decline within the first day of holding, and a further, smaller, decline on the second day. Plasma FSH was less labile and in several cases, remained high even when $\mathrm{T}$ dropped (e.g., Fig. 4B) or tended to recover while $T$ remained depressed (e.g., Fig. 4B). $T_{4}$ was also more variable than $\mathrm{T}$ and although there was the same general tendency to decline, it tended to fall more slowly or even remain relatively stable in the face of declining $\mathrm{T}$.

\section{DISCUSSION}

The present observations on hormone levels in painted turtles under natural conditions differ in several ways from those presented in earlier reports of seasonal cycles in this species and help resolve previous discrepancies between seasonal cycles and laboratory experimental studies. Moreover, these data provide new information for evaluating the relationship of testicular activity to gonadotropin levels and thyroidal function. Although direct measurements of testicular weight and histology could not be made here, this information is available from several previous studies of northern C. picta (Moll, 1973), including one at a nearby study site in southwestern Michigan (Gibbons, 1968). These studies showed that testicular growth commenced in mid to late July and peaked in late August; thus, most of testicular growth was completed shortly before the second peak in plasma androgen was attained. Testes diminish in size with the cessation of spermatogenesis and the evacuation of sperm into the epididymides in 


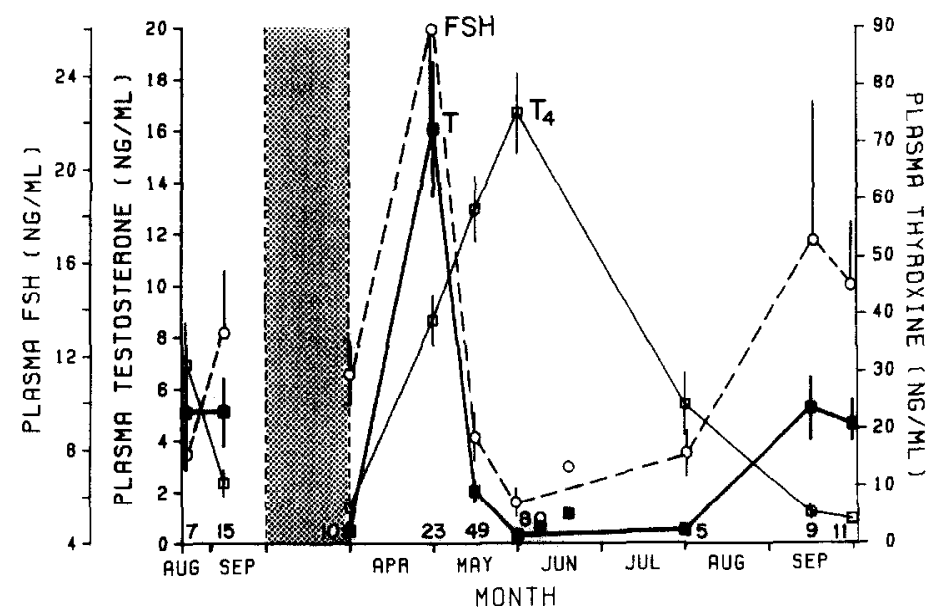

Fig. 3. Mean biweekly values for plasma FSH (open circles, dashed curve), testosterone (T, shaded squares) and thyroxine ( $\mathrm{T}_{4}$, open squares) in adult male Chrysemys picta sampled immediately after capture in the field. Vertical lines show one standard error. The stippled area between September and April represents the period of winter hibernation. Samples sizes are shown above the abscissa for each date. Testicular recrudescence and spermatogenetic activity normally begins in mid July and peaks in late August.

late September-October, shortly before the onset of hibernation (see also Ganzhorn and Licht, 1983). The interstitial cell cycle

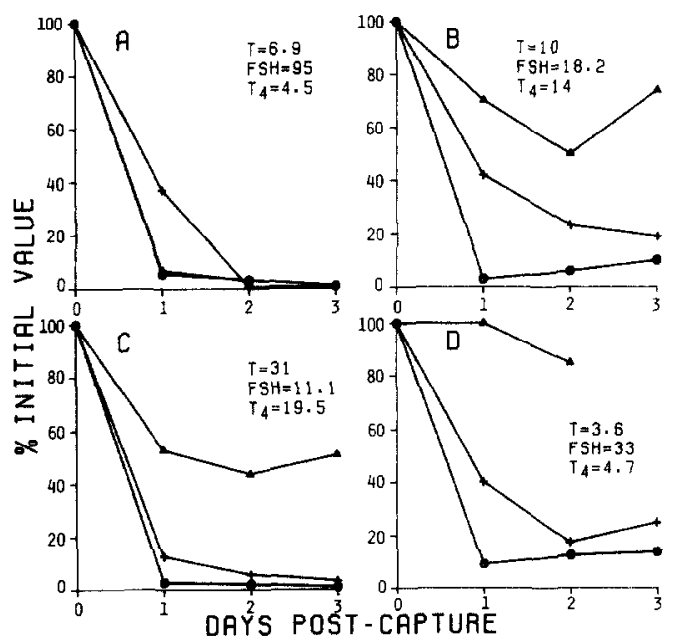

FIG. 4. Postcapture changes in plasma hormones in four male Chrysemys picta: (A) $\mathrm{FSH}$; (O) $\mathrm{T} ;(+) \mathrm{T}_{4}$. Animals were placed in aquatic pens immediately after the first blood sample was taken and then resampled daily. Starting plasma levels $(\mathrm{ng} / \mathrm{ml}$ ) of each hormone (at Day 0) are shown by data in insets and values for subsequent days are expressed as a percentage of these values. Dates were: A, September 13; B, September 10; C, April 26; D, September 11. is typical of other temperate chelonians with the Leydig cells being most conspicuous in spring (Moll, 1973; Callard et al., 1976), although as mentioned earlier, the value of the typical histological assessment of Leydig cells as an indicator of testicular steroidogenic activity in this species has been questioned (Ganzhorn and Licht, 1983).

The present data for freshly captured turtles supports earlier reports (Callard et al., 1976; Ganzhorn and Licht, 1983) that plasma androgen is maximal in early spring coincident with minimal activity in the seminiferous tubules. However, several notable discrepancies are evident between these data and previous findings. First, the present data demonstrate that plasma androgen levels are biphasic and are again elevated to almost the same levels in fall in association with testicular growth and spermatogenetic recrudescence as was postulated from experimental studies (Ganzhorn and Licht, 1983). This biphasic annual pattern is similar to that reported for the tortoise, Testudo hermani (Kuchling et al., 1981), but distinct from the monophasic an- 


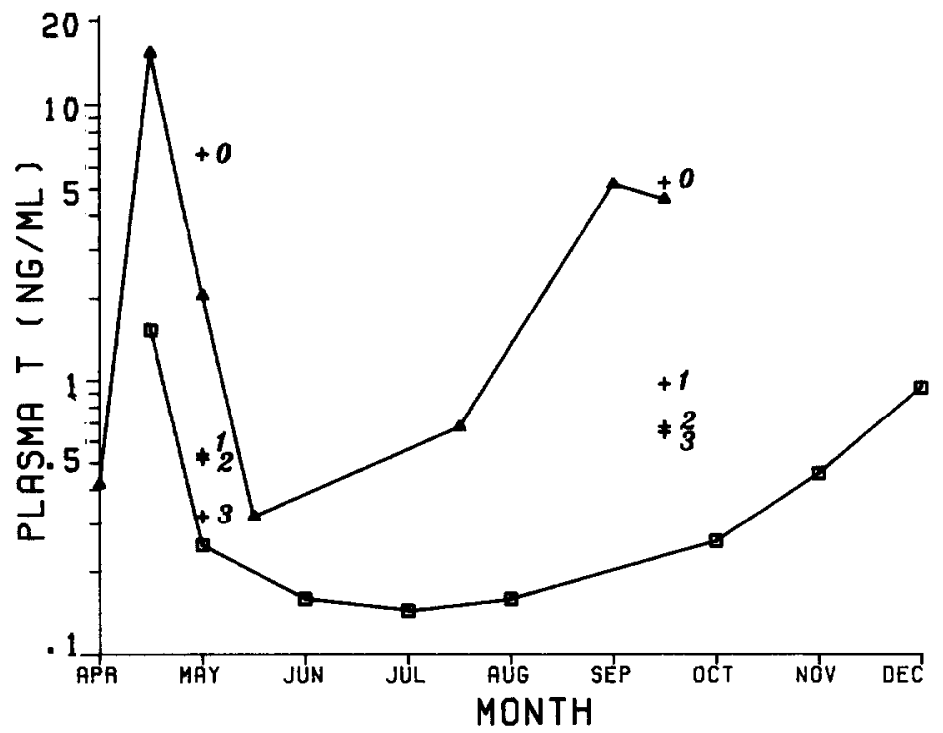

FIG. 5. Comparison of data for annual cycles in plasma $\mathrm{T}$ in adult male Chrysemys picta reported here and in a previous study. The lower curve $(\square)$ is based on data from the study of Callard $e t$ al. (1976) and the upper curve ( $\boldsymbol{\Lambda})$ is based on data from freshly captured animals in this study (biweekly data from Fig. 3). A sample of 8 animals captured in early May and 16 animals in late September were sampled for 3 days after capture; mean values for the initial sample (0) and each subsequent day $(1,2$, and 3$)$ are shown by an "+" next to the corresponding sample day.

drogen cycles (with only fall or spring peaks) reported for several other chelonians (Licht, 1982; Licht et al., 1984), including the more subtropical congener, $C$. dorbigni (Silva et al., 1984).

Second, the average level of plasma $T$ observed at the spring peak in the previous study (Callard et al., 1976) was about an order of magnitude below that observed here; in fact, it was significantly lower than the fall peak observed in this study (Fig. 5). Also, it was previously reported that $T$ levels began to increase in November-December, suggesting that some increment in hormone titers might occur during hibernation (these data were presumably from animals held over winter in supply houses or the laboratory, probably under unnatural seasonal conditions). In contrast, data for freshly captured turtles indicate that androgens drop during hibernation and are minimal when animals emerge in spring (Fig. 2).

Several explanations are suggested for discrepancies between this and previous studies. First, it is evident that the spring peak in $T$ is brief and hence a single monthly sample might well fail to reflect its true magnitude. The transitory nature of the spring rise may also explain why consistently higher levels were observed in $C$. picta in April of one year than in the next (Ganzhorn and Licht, 1983); and also why Silva et al. (1984) failed to observe a distinct spring peak in the subtropical $C$. dorbigni. Equally likely is that the previous studies on Chrysemys reflected the "stress" of captivity.

Both the reproductive and thyroid hormones in the painted turtle are clearly susceptible to the effects of capturing and handling in much the same way as was shown for the bullfrog (Licht et al., 1983). The likelihood that such effects are important in accounting for discrepancies among studies is illustrated by comparing the previously published values on plasma $T$ with those obtained on turtles that were sampled at the 
time of collection and after 1-3 days of confinement (Fig. 5). Tests performed here do not distinguish between the effects of capture/captivity and those induced by the initial blood sampling procedure, but similar studies in bullfrogs demonstrated that the depression of hormone levels is related to removing the animal from its natural habitat rather than blood sampling per se (Licht et al., 1983). Although we were concerned that some turtles collected in baited traps might be stressed, since the animals might be confined for up to 2 days, this group was not clearly different from animals captured by other methods in this study. Turtles in these traps may be "unaware" of having been trapped, perhaps because they remain undisturbed and in their normal area of activity. Thus, endocrine studies involving the reproductive or thyroid axis based on commercially collected turtles must be viewed with caution. Even samples taken within a day of capture may be unsatisfactory for evaluating normal endogenous levels. These results raise further concern about several of the published studies on turtles and squamates that were based on recently captured animals (e.g., Bona-Gallo et al., 1980; reviewed in Licht, 1982). The mechanisms for these "stress" effects is unclear; gonadotropin appeared to be less consistently affected than was androgen.

The physiological significance of the seasonal changes in $T$ and $T_{4}$ are not yet known. The physiological role of $\mathrm{T}_{4}$ in chelonians is too little studied to allow speculations about the importance of its pronounced seasonal cycle. Although direct experimental evidence for the role of $\mathrm{T}$ in chelonian sex behavior is limited, it has been assumed that the spring elevation in $T$ relates to mating behavior. Unfortunately, there are actually few quantitative data on the timing of mating activity in this species. Limited observations summarized by Gibbons (1968) indicate that mating activity does occur in both spring and fall, but the relative intensity of sex behavior in the two periods is difficult to assess. Nonetheless, it appears that both peaks in plasma $\mathrm{T}$ may be related to sex behavior. Even though the spring mating period appears to continue beyond the time when plasma $T$ is elevated (Gibbons, 1968), studies in the green sea turtle suggest that the transient peak in $T$ could have important consequences on subsequent mating activity (Licht et al., 1985). On the other hand, plasma $T$ appeared to be minimal around the time of spring mating in the subtropical C. dorbigni (Silva et al., 1984); unfortunately, the infrequent sampling schedule, uncertainty about handling methods, and imprecise data on mating activity raises concern about these findings.

The reciprocal relationship between plasma androgen and $T_{4}$ in spring is like that reported for the cobra (Bona-Gallo et $a l ., 1980$ ) and reminiscent of the situation described in several avian species in which androgen and $\mathrm{T}_{4}$ may have an inhibitory effect on the timing of one another (Jallageas and Assenmacher, 1979). A variety of studies have implicated the thyroid hormones in reproductive function in squamate reptiles (turtles have not been tested), but the nature of this relationship is unclear since both hypo and hyperthyroidal conditions tend to inhibit gonadal activity (Licht, 1984). Experimental verification for such a reciprocal physiological relationship between the thyroid and gonadal cycles is lacking for reptiles and it should be noted that this pattern was not observed in the green sea turtle (Licht et al., 1985). Thus, separate androgen and thyroid cycles may simply reflect independent or differential responsiveness of the gonads and thyroid to changing environmental stimuli in the temperate-zone reptiles.

A major difference in environmental temperatures is evident between the present situation and that for the sea turtle; temperatures varied by only a few degrees daily and annually in the sea turtle study. Although temperature relations for the 
painted turtle are more complex than for the almost strictly aquatic sea turtle, the painted turtle clearly undergoes profound daily and annual temperature changes and these confound interpretation of hormonal profiles. For example, the rapid rise in temperature after emergence from hibernation may act as a potent stimulus to the activation of the gonadal and thyroid axes (although the two may not necessarily respond equally). The high levels of androgen and FSH in early spring occur at lower average daily body temperatures than in fall and differences in turnover of hormones may account for the somewhat higher average levels attained in spring. These thermal considerations may also be important for understanding the seasonal dissociation between androgen production and testicular growth/spermatogenesis.

The high correlation between plasma FSH and T supports physiological studies (with heterologous hormones) that indicated that for some turtles, FSH was even more effective than LH in stimulating androgen secretion (Licht, 1979). However, the correlation between FSH and T is less clear for individuals sampled after capture; i.e., $\mathrm{T}$ sometimes dropped faster or to a larger extent than did FSH (e.g., Fig. 4B, D); other factors associated with "stress", may alter the relationship between these hormones. Perhaps more surprising is the failure to observe an elevation in FSH when gonial proliferation begins in late spring ( $\sim$ May) or when spermatogenesis and testicular growth begin (July-August) (Moll, 1973). The pattern represented by the curve drawn for FSH in Fig. 3 may be somewhat misleading because of the dearth of data for early summer; i.e., the rise between late July and mid-September may have becn more rapid than indicated, since $\mathrm{FSH}$ increased to maximum within 2 weeks in April (Fig. 3). Indeed, although not statistically significant, there is a slight rise in mean FSH between the end of May and the end of July; in fact, we cannot rule out the possibility of another peak in early July.

A related issue concerns the lack of appreciable spermatogenetic activity in April when plasma FSH is maximal. This situation can be explained from experimental studies on this species and various physiological studies in other reptiles (e.g., Licht, 1975). In particular, it has been shown that warm temperatures are required for both testicular growth and androgen secretion to respond to elevated gonadotropin; however, the former is a much slower and more temperature-sensitive process than is the latter. While days or even weeks of gonadotropin stimulation at warm body temperatures are required for testicular growth, once Leydig cells are hypertrophied, androgen secretion may respond to gonadotropin treatment within minutes. It appears that Leydig cells are developed in fall and remain responsive until spring. The period following emergence from hibernation in April is characterized by very low nocturnal temperatures and days that are still relatively cool and short (hence limited opportunity for basking). Therefore, it is likely that temperatures are neither warm enough nor is FSH elevated long enough for testicular growth to begin, even though the reproductive system of the painted turtle is capable of being activated in spring (Ganzhorn and Licht, 1983). By the time temperatures are elevated sufficiently, FSH has dropped (June-July) and testes do not grow again until late summer when the second elevation is FSH occurs during a warmer period. Thus, there is at present no basis for the hypothesis of separate FSH and LH cycles underlying a seasonal dissociation between spermatogenesis and androgen secretion.

Temperate-zone turtles typically show a single relatively brief period of spermatogenesis with complete evacuation of sperm. The cessation of spermatogenesis before hibernation (e.g., late August-September) is 
somewhat unexpected in light of continuously elevated FSH during this period. Either testes become refractory to gonadotropin or some other factors intervene to alter their responsiveness. Data for the cobra (Bona-Gallo et al., 1980) suggested that gonadal regression which also occurred in the face of elevated gonadotropin might be caused by the coincident rise in $\mathrm{T}_{4}$ as has been proposed for some birds. While such a reciprocal relationship between thyroidal and gonadal activity might be important in the spring, this type of interaction would not explain spermatogenetic regression in fall since $T_{4}$ is minimal in the turtle. We were, unfortunately, unable to measure $T_{3}$.

We recognize that caution is required in interpreting single daily samples of circulating hormones since mammalian data have demonstrated both short-term pulsatile release and diurnal fluctuations in these hormones. Studies in the green sea turtle indicated a much more constant pattern, at least in $\mathrm{T}_{4}$ and $\mathrm{T}$, with no discernible diurnal cycle at any time of the annual cycle (Licht et al., 1984). However, the sea turtle did not experience the large diurnal fluctuation likely to occur in certain seasons in the painted turtle. Thus, we must consider the possibility that different diurnal patterns as well as gross seasonal changes may occur in the latter species. Studies on the sea turtle demonstrate that major seasonal temperature changes are not required to induce a pronounced annual cycle in reproductive hormones and since painted turtles kept at constant warm temperatures still tend to exhibit cycles in testicular size (Ganzhorn and Licht, 1983), it is possible that the annual reproductive cycle has an endogenous component that is partially independent of external cues. The existence of large seasonal fluctuations in body temperature complicates interpretation of the mechanisms and consequences of seasonal patterns in plasma hormones since it is not yet possible to distinguish between changes in secretion vs turnover rates, and as discussed earlier, variability in responsiveness of target organs can alter the effectiveness of a given titer of hormone. These data illustrate the dynamic nature of the seasonal changes in the turtle and indicate that future studies should consider frequent sampling protocols and sampling from undisturbed animals.

\section{ACKNOWLEDGMENTS}

As we thank Raymond Pang for technical assistance in the analysis of hormone samples and Tal Novak, Mark Hutchison, and Patty Orleans for assistance with data collection. Jack Burch, UMMZ, provided a centrifuge for use in these experiments. This work was supported in part from grants from the National Science Foundation to P.L. (PCM-8109846) and to J.C. (DEB 79-06031). Data analysis and manuscript preparation was aided by contract DE-AC09-SR00-819 between the University of Georgia and the United States Department of Energy.

\section{REFERENCES}

Bona-Gallo, A., Licht, P., MacKenzie, D. S., and Lofts, B. (1980). Annual cycles in levels of pituitary and plasma gonadotropin, gonadal steroids, and thyroid activity in the Chinese cobra (Naja naja). Gen. Comp. Endocrinol. 42, 477-493.

Callard, I. P., Callard, G. V., Lance, V., and Eccles, S. (1976). Seasonal changes in testicular structure and function and the effects of gonadotropins in the freshwater turtle, Chrysemys picta. Gen. Comp. Endocrinol. 30, 347-356.

Congdon, J. D., and Tinkle, D. W. (1982). Reproductive energetics of the painted turtle (Chrysemys picta). Herpetologica 38, 228-237.

Ganzhorn, D., and Licht, P. (1983). Regulation of seasonal gonadal cycles by temperature in the painted turtle Chrysemys picta. Copeia 1983 , 347-358.

Gibbons, J. W. (1968). Reproductive potential, activity and cycles in the painted turtle, Chrysemys picta. Ecology 49, 400-409.

Jallageas, M., and Assenmacher, I. (1979). Further evidence for reciprocal interactions between the annual sexual and thyroid cycles in male Peking ducks. Gen. Comp. Endocrinol. 37, 44-51.

Kuchling, G. R., Skolek-Winnishch, R., and Bamber, E. (1981). Histochemical and biochemical investigation on the annual cycle of testis, epididymis, and plasma testosterone of the tortoise, Testudo 
hermanni hermanni Gmelin. Gen. Comp. Endocrinol. 44, 194-201.

Licht, P. (1975). Temperature dependence of the actions of mammalian and reptilian gonadotropins in a lizard. Comp. Biochem. Physiol. 50A, 221222.

Licht, P. (1978). Studies on the immunochemical relatedness among tetrapod gonadotropins and their subunits with antisera to sea turtle hormones. Gen. Comp. Endocrinol. 36, 68-78.

Licht, P. (1979). Reproductive endocrinology of reptiles and amphibians. Annu. Rev. Physiol. 41, $337-351$.

Licht, P. (1982). Endocrine patterns in the reproductive cycles of turtles. Herpetologica 38, 51-61.

Licht, P. (1984). Reptiles. In "Marshall's Physiology of Reproduction" (G. E. Lamming, ed.), pp. 206282. Churchill, Edinburgh.

Licht, P., and Papkoff, H. (1985). Reevaluation of the relative activities of the pituitary glycoprotein hormones (FSH, LH, and TSH) from the green sea turtle, Chelonia mydas. Gen. Comp. Endocrinol. 58, 443-451.

Licht, P., MacKenzie, D. S., Papkoff, H., and Farmer, S. W. (1977). Immunological studies with the gonadotropins and their subunits from the green sea turtle Chelonia mydas. Gen. Comp. Endocrinol. 33, 231-241.

Licht, P., McCreery, B. R., Barnes, R., and Pang, R. (1983). Seasonal and stress related changes in plasma gonadotropins, sex steroids and corticosterone in the bullfrog, Rana catesbeinana Gen. Comp. Endocrinol. 50, 124-145.

Licht, P., Wood, J. F., and Wood, F. E. (1985). Annual and diurnal cycles in plasma testosterone and thyroxine in the male green sea turtle Chelonia mydas. Gen. Comp. Endocrinol. 57, 335-344.

Moll, E. O. (1973). Latitudinal and interspecific variation in reproduction of the painted turtle, Chrysemys picta. Herpetologica 29, 307-318.

Moll, E. O. (1979). Reproductive cycles and adaptations. In "Turtles: Perspective and Research" $M$. Harless and H. Morlock, eds.), pp. 305-331. Wiley, New York.

Silva, A. M. R., Moraes, G. S., and Wasserman, G. F. (1984). Seasonal variations of testicular morphology and plasma levels of testosterone in the turtle Chrysemys dorbigni. Comp. Biochem. Physiol. 78A, 153-157.

Tinkle, D. W., Congdon, J. D., and Rosen, P. C. (1981). Nesting frequency and success: Implications for the demography of painted turtles. Ecology 62, 1426-1432. 